\title{
CINÉTICA DA OXICOMBUSTÃO DO CARVÃO DA MINA DE CANDIOTA - RS COM ALTO TEOR DE CINZAS
}

\author{
K. G. P. NUNES ${ }^{1}$, P. J. MELO ${ }^{1}$, E. OSÓRIO², N. R. MARCÍLIO ${ }^{1}$ \\ ${ }^{1}$ Universidade Federal do Rio Grande do Sul, Departamento de Engenharia Química \\ ${ }^{2}$ Universidade Federal do Rio Grande do Sul, Departamento de Minas, Metalúrgica e Materiais \\ E-mail: keilagpn@gmail.com
}

\begin{abstract}
RESUMO - O trabalho apresenta o estudo cinético da reação de oxicombustão do carvão mineral extraído da mina de Candiota/RS, com alto teor de cinzas. Na tecnologia da oxicombustão a queima ocorre com uma mistura de $\mathrm{O}_{2}$ com alto teor de $\mathrm{CO}_{2}$. Os experimentos foram realizados em termobalança nas temperaturas de $600{ }^{\circ} \mathrm{C}$ a $900{ }^{\circ} \mathrm{C}$, em pressão atmosférica, com concentrações de $\mathrm{O}_{2}$ variando entre 10 e $30 \% \mathrm{v} / \mathrm{v}$ em $\mathrm{CO}_{2}$. Primeiramente, se determinou a temperatura de pirólise do carvão a fim de eliminar os compostos voláteis, evitando assim a sua influência na reação de oxicombustão. Os resultados experimentais foram tratados matematicamente com modelos retirados da literatura para determinação dos parâmetros cinéticos, como energia de ativação e ordem de reação.
\end{abstract}

\section{INTRODUÇÃO}

O Brasil é responsável pelo lançamento de 2 bilhões de toneladas de $\mathrm{CO}_{2}$ na atmosfera anualmente. Esse índice corresponde a 5 \% das emissões mundiais desse gás, deixando o Brasil entre os 5 países que mais poluem (BEN, 2013).

As emissões de $\mathrm{CO}_{2}$ correspondem a $80 \%$ do total dos gases emitidos, por isso as pesquisas para a captura o e armazenamento se concentram sobre esse gás (IEA, 2013). A oxicombustão de carvão mineral vem sendo estudada como alternativa tecnológica devido à facilidade de captação integral de $\mathrm{CO}_{2}$ resultante da combustão, bem como a captação de outros poluentes, de forma a permitir seu uso de forma pouco nociva ao meio. A técnica consiste na utilização de uma corrente de oxigênio diluído pelos gases exaustos reciclados como comburente, produzindo um gás rico em $\mathrm{CO}_{2}$, facilitando assim a sua captura (TOFTEGAARD et al., 2010).

No Brasil, o estudo cinético da gaseificação de carvão iniciou na década de 1980 com Schmal et al. (1982) e José H. J. (1989). Atualmente, as pesquisas sobre oxicombustão estão direcionadas a caracterização, modelagem e análise cinética em leito fluidizado (DUAN et al, 2014; LECKNER, et al. 2014; ROY and BHATTACHARYA, 2014).

A aquisição permanente de dados sobre a combustão do carvão mineral nessa nova condição de operação é necessária para o dimensionamento ou adaptação de equipamentos a uma 
forma de produção energética mais limpa, acompanhando a evolução das legislações ambientais cada vez mais restritivas quanto uso de combustíveis fósseis.

O alto teor de cinzas presente nos carvões brasileiros faz com que os processos de beneficiamento tenham custo elevado especialmente para seu uso energético. Como representam a maior reserva energética do Brasil, é constante a busca por processos cada vez mais eficientes, técnica e economicamente, para seu uso energético.

O carvão utilizado para o estudo termogravimétrico em condições de oxicombustão foi coletado da mina de Candiota, localizada no município de Candiota no Rio Grande do Sul. Os experimentos foram realizados utilizando concentrações de $\mathrm{O}_{2}$ em $\mathrm{CO}_{2}$ entre $10 \%$ e $30 \%$ e temperaturas variando de $600{ }^{\circ} \mathrm{C}$ a $900{ }^{\circ} \mathrm{C}$.

\section{MATERIAIS E MÉTODOS}

\subsection{Produção de char}

O char, produto da desvolatilização do carvão na ausência de ar, foi preparado em atmosfera de $\mathrm{N}_{2}$, a partir de três faixas granulométricas de carvão: $\mathrm{A}(0,5$ a $0,85 \mathrm{~mm}), \mathrm{B}(0,85$ a $1,00 \mathrm{~mm})$ e $\mathrm{C}(1,68$ a 2,00 $\mathrm{mm})$. Diferentes granulometrias foram utilizadas para que fosse observado o efeito dessas no tamanho dos poros e na área superficial do char.

Cerca de 150 g foram colocadas em um reator de aço inox, descrito em Nunes e Marcílio (2012). A amostra permaneceu 60 minutos na temperatura pré-determinada. Utilizaram-se duas temperaturas para desvolatilização, $880{ }^{\circ} \mathrm{C}$ e $900{ }^{\circ} \mathrm{C}$ para observar a quantidade de matéria volátil remanescente no char e seu possível efeito sobre a estrutura do char produzido. Com base nesses resultados selecionou-se a temperatura para as reações de oxicombustão.

As amostras produzidas foram submetidas às análises imediata, elementar, diâmetro de poro e área superficial. Os resultados se encontram na Tabela 1.

\subsection{Oxicombustão}

Os experimentos de oxicombustão do char foram conduzidos em uma termobalança a pressão atmosférica, utilizando-se aproximadamente $30 \mathrm{mg}$ de char num cadinho tipo prato. Um termopar localizado abaixo do prato monitorava a temperatura, que variou de 600 a $900{ }^{\circ} \mathrm{C}$. Os gases utilizados nos experimentos foram introduzidos na termobalança a uma taxa de 125 mL.min. ${ }^{-1}$, com concentrações de $\mathrm{O}_{2}$ em $\mathrm{CO}_{2}$ variando de 10 a 30 \%. A conversão $X$ do char foi calculada segundo a equação 1.

$$
X=\frac{M_{i}-M}{M_{i}-M_{C}}
$$

Onde $M_{i}$ representa a massa inicial de char $(\mathrm{mg}), M$ a massa instantânea $(\mathrm{mg}), M_{c}$ a massa 
após a reação de oxicombustão (mg).

Para o tratamento e discussão dos dados cinéticos obtidos utilizou-se o modelo matemático do núcleo não reagido, que considera que a reação se inicia na superfície externa à partícula e se move em direção ao centro do sólido deixando para trás uma camada de sólido inerte, ou seja, as cinzas. A equação matemática em função da conversão ao longo do tempo será apresentada durante a discussão dos resultados.

\section{RESULTADOS E DISCUSSÕES}

\subsection{Efeito da temperatura de pirólise}

Uma amostra contendo cerca de $3 \mathrm{~kg}$ de carvão beneficiado foi submetida a análise imediata e elementar e sua composição encontra-se na Tabela 1.

\begin{tabular}{l|l}
\multicolumn{2}{c}{ Tabela 1 Composição do carvão } \\
\hline Análises & Teores (\%) \\
\hline Análise imediata1 $^{1}$ & \\
Matéria volátil & 21,1 \\
Cinzas & 52,8 \\
Carbono fixo & 26,1 \\
\hline Análise elementar ${ }^{2}$ & \\
$\mathrm{C}$ & 74,85 \\
$\mathrm{~N}$ & 1,50 \\
$\mathrm{~S}$ & 3,40 \\
$\mathrm{H}$ & 8,25 \\
$\mathrm{O}$ & 12,00 \\
\hline${ }^{2}$ base seca \\
${ }^{2}$ base seca isenta de cinzas (dados a serem confirmados)
\end{tabular}

Para avaliar o efeito da estrutura do char após a pirólise, utilizaram-se duas temperaturas. Com as faixas granulométricas A e C procedeu-se a pirólise a $900{ }^{\circ} \mathrm{C}$ e com a faixa granulométrica $\mathrm{B}$, a desvolatilização ocorreu a $880^{\circ} \mathrm{C}$. Os teores de matéria volátil remanescente nas amostras, a área superficial e o diâmetro do poro resultante encontram-se na Tabela 2.

Tabela 2: Caracterização dos chars produzidos com diferentes temperaturas

\begin{tabular}{c|c|c|c|c|c}
\hline Char & $\begin{array}{c}\text { Temperatura } \\
\left({ }^{\circ} \mathrm{C}\right)\end{array}$ & $\begin{array}{c}\text { Granulometria } \\
(\mathrm{mm})\end{array}$ & $\begin{array}{c}\text { Matéria volátil } \\
(\%)\end{array}$ & $\begin{array}{c}\text { Área superficial }- \\
\mathrm{BET}\left(\mathrm{m}^{2} \cdot \mathrm{g}^{-1}\right)\end{array}$ & $\begin{array}{c}\text { Diâmetro do poro } \\
(\mathrm{nm})\end{array}$ \\
\hline $\mathrm{A}$ & 900 & $0,5-0,85$ & 1,23 & 95,852 & 1,091 \\
$\mathrm{~B}$ & 880 & $0,85-1,00$ & 1,47 & 91,818 & 1,024 \\
C & 900 & $1,68-2,00$ & 1,34 & 94,114 & 0,970 \\
\hline
\end{tabular}

Observando os resultados apresentados na Tabela 2, para uma mesma temperatura de pirólise, o teor de matéria volátil liberado é ligeiramente maior para um char de granulometria 
inferior. Também é possível observar que partículas menores tem maior área superficial e poros com diâmetros maiores disponíveis para reação. O efeito da matéria volátil presente no char durante a oxicombustão é mais pronunciado quando observamos seu comportamento em termobalança, como é mostrado na Figura 1.

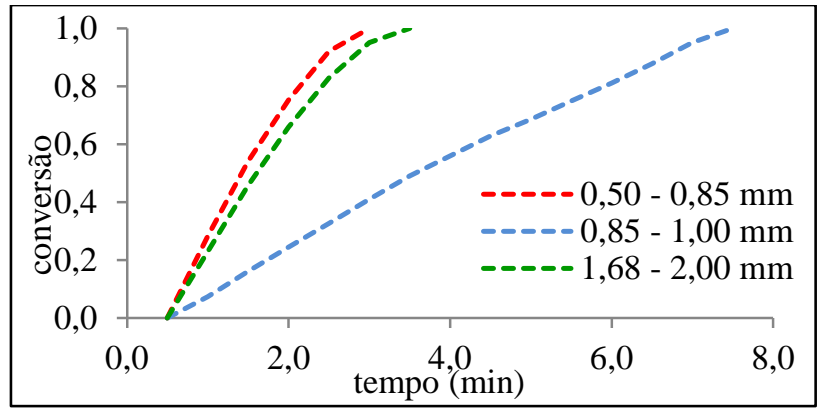

Figura 1 Taxa de conversão para as granulometrias A, B e C com $10 \%$ de $\mathrm{O}_{2}$ em $\mathrm{CO}_{2}$.

Nesta figura observa-se que os chars pirolisados a $900^{\circ} \mathrm{C}$, apresentam uma alta taxa de conversão, enquanto que o char pirolisado a $880^{\circ} \mathrm{C}$, por ainda possuir uma maior quantidade de matéria volátil no seu interior, reage mais lentamente nas condições de oxicombustão.

Com base no resultado apresentado, selecionou-se a granulometria A (0,50-0,85 mm) para dar continuidade aos ensaios termogravimétricos em condição de oxicombustão. Ao utilizar uma partícula de menor tamanho minimizam-se os efeitos difusivos, podendo considerar para fins de cálculo somente a reação química.

\subsection{Efeito da temperatura de oxicombustão}

A fim de avaliar o efeito da temperatura sobre a taxa de reação de oxicombustão do char utilizou-se temperaturas de $600{ }^{\circ} \mathrm{C}, 700{ }^{\circ} \mathrm{C}, 800{ }^{\circ} \mathrm{C}$ e $900{ }^{\circ} \mathrm{C}$, fixando as demais variáveis. Estas temperaturas foram escolhidas em função dos resultados preliminares obtidos no ensaio termogravimétrico, mostrado na Figura 2.

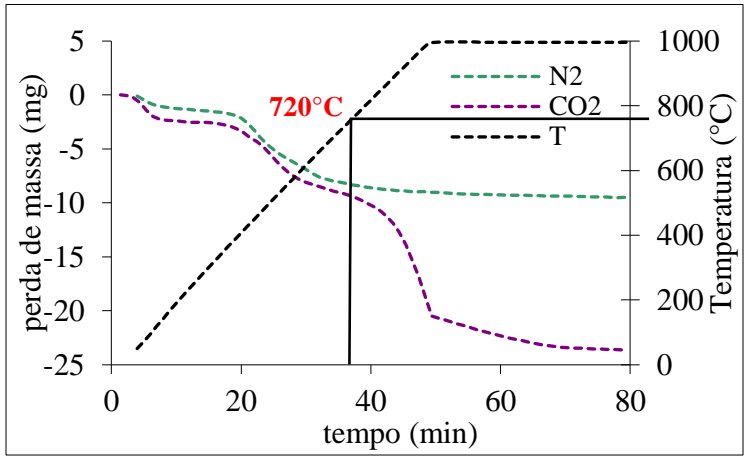

Figura 2 Perda de massa do carvão em função do tempo (granulometria B: 0,85 - 1,00 mm).

Conforme se observa na Figura 2 o consumo de carbono, ou seja, a reação de Boudouard inicia-se próximo aos $720{ }^{\circ} \mathrm{C}$, ponto em que as curvas passam a ter comportamento distinto. 
Portanto selecionou-se temperaturas anteriores a reação de consumo de carbono e posterior para observarmos esse efeito na reação de oxicombustão.

A Figura 3, a seguir, mostra as curvas de conversão do char em função do tempo para as temperaturas de $600{ }^{\circ} \mathrm{C}, 700{ }^{\circ} \mathrm{C}, 800{ }^{\circ} \mathrm{C}$ e $900{ }^{\circ} \mathrm{C}$, utilizando como gás reagente uma mistura 30 $\% \mathrm{O}_{2} / 70 \% \mathrm{CO}_{2} \mathrm{v} / \mathrm{v}$.

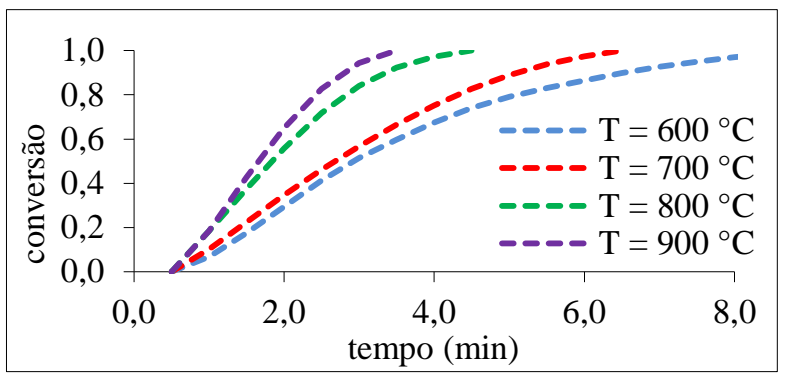

Figura 3 Taxa de reação em função da temperatura para a mistura $30 \% \mathrm{O}_{2} / 70 \% \mathrm{CO}_{2}$.

Observa-se na Figura 3 que aumentando a temperatura da reação, a taxa de conversão do char se intensifica. Este comportamento pode ser um indicativo de que o regime cinético controla o processo. A temperatura de oxicombustão foi limitada a $900{ }^{\circ} \mathrm{C}$, pois como já foi observado por Pohlmann et al (2010) e Nunes e Marcílio (2012), por volta de $1000{ }^{\circ} \mathrm{C}$ ocorre o predomínio do controle reacional misto.

\subsection{Influência da concentração de $\mathrm{O}_{2}$ na mistura gasosa}

Segundo Khatami, et al. (2012), para se obter, em condições de oxicombustão, o mesmo perfil de temperaturas de combustão em ar atmosférico deve-se usar uma mistura reagente contendo $30 \%$ de $\mathrm{O}_{2}$ em $\mathrm{CO}_{2}$. Neste trabalho, além de utilizar essa concentração recomendada, utilizou-se também concentrações de $10 \%$ e $20 \%$ de $\mathrm{O}_{2}$ em $\mathrm{CO}_{2}$ a fim de determinar o fenômeno químico que rege a reação. A Figura 4 apresenta a curva de conversão do char em diferentes concentrações de $\mathrm{O}_{2}$ na temperatura de $600^{\circ} \mathrm{C}$.

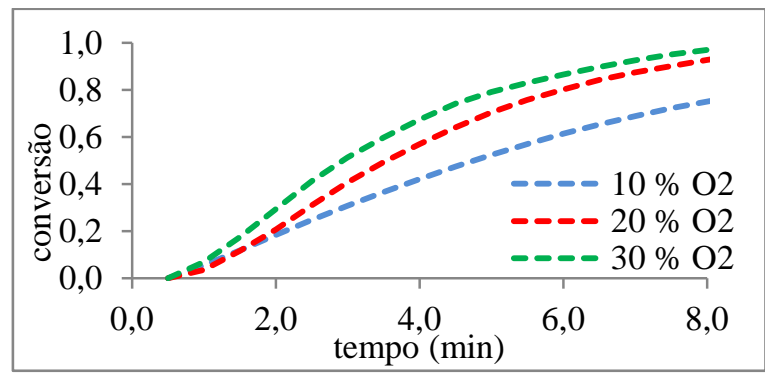

Figura 4 Conversão de char versus o tempo para diferentes concentrações de mistura $\mathrm{O}_{2} / \mathrm{CO}_{2}$ a $600^{\circ} \mathrm{C}$.

Observa-se que o aumento na concentração de $\mathrm{O}_{2}$ na mistura gasosa aumenta a velocidade de conversão do char. Para as demais concentrações o mesmo efeito também é observado. Esse fenômeno indica que o processo é determinado pela reação química. 


\subsection{Modelo cinético - Modelo do Núcleo não Reagido}

A Figura 5 apresenta a tentativa de ajustar o modelo do núcleo não reagido aos dados experimentais em três situações: (a) quando a difusão do reagente na camada de cinzas controla o processo, (b) quando a reação química controla o processo e (c) quando a difusão na camada gasosa externa à partícula controla o processo.

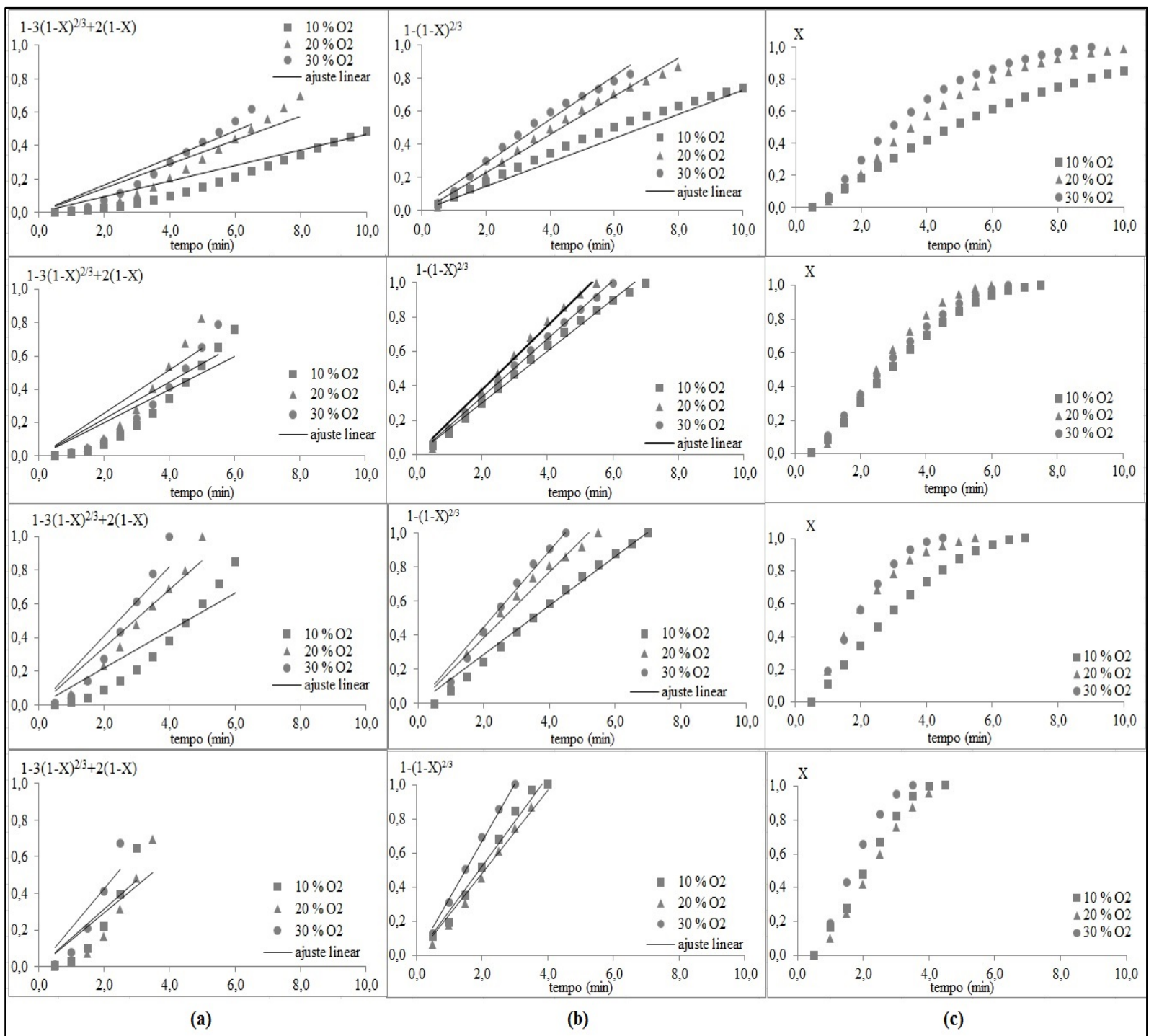

Figura 5 Ajuste do modelo do núcleo não reagido aos resultados experimentais quando: a) quando a difusão do reagente na camada de cinzas controla o processo, b) quando a reação química controla o processo e c) quando a difusão na camada gasosa externa à partícula controla o processo, para as temperaturas de $600{ }^{\circ} \mathrm{C}, 700^{\circ} \mathrm{C}, 800^{\circ} \mathrm{C}$ e $900{ }^{\circ} \mathrm{C}$, respectivamente. 
Conforme se observa na Figura 5a e 5c, não há um bom ajuste do modelo aos dados experimentais, pois os modelos não reproduzem adequadamente as medidas realizadas. Na Figura 5b o ajuste do modelo do núcleo não reagido aos dados experimentais foi realizado considerando o regime cinético como a etapa controladora. Verifica-se, neste caso, que pelo bom ajuste do modelo aos dados experimentais, a reação química seja a etapa determinante do processo reacional.

A Figura 6 apresenta uma amostra de char sobre o cadinho de reação antes e após a reação de oxicombustão em termobalança. Devido a grande quantidade de cinzas na matriz carbonosa, não há uma redução significativa no tamanho da partícula, justificando, assim, o uso do modelo do núcleo não reagido para o tratamento matemático dos dados experimentais.

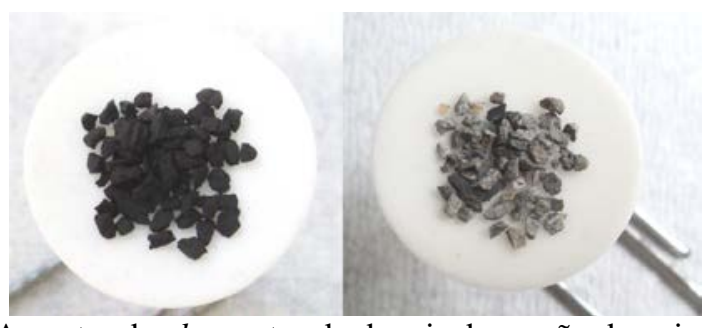

Figura 6 Amostra de char antes de depois da reação de oxicombustão.

Nesse caso, utilizando o modelo do núcleo não reagido com controle cinético determinando o sistema, o tempo $(\tau)$ para a conversão completa da partícula é dado pela equação (2):

$$
\tau=\frac{\rho R}{k P_{O_{2}}}
$$

Onde $\rho$ é a massa específica da amostra $\left(\mathrm{g} \cdot \mathrm{cm}^{-3}\right), R$ é o raio médio da partícula $(\mathrm{cm})$ e $k$ é a velocidade específica da reação $\left(\mathrm{gmol}^{-1} \cdot \mathrm{min}^{-1} \cdot \mathrm{cm}^{-2} \cdot \mathrm{atm}^{-\mathrm{n}}\right)$ e $P_{\mathrm{O}_{2}}$ é a pressão parcial de $\mathrm{O}_{2}(\mathrm{~atm})$.

Supondo que a taxa da reação obedeça a equação (3):

$$
\left(-r_{A}\right)=k P_{O_{2}}^{n}
$$

Portanto, a taxa de reação $\left(-r_{A}\right)$ pode ser calculada através da equação (4), ou seja:

$$
\left(-r_{A}\right)=\frac{R \cdot \rho}{\tau}
$$

Onde $R=0,03375 \mathrm{~cm}, \rho=1,96 \mathrm{~g} \cdot \mathrm{cm}^{-3}$ e $\tau=$ valores experimentais obtidos a partir dos gráficos de conversão, como o da Figura 4.

Com a equação (3), determinam-se os valores de $k$ e a ordem da reação, $n$. Utilizando a equação de Arrhenius (equação 5) calcula-se, então, o fator pré-exponencial, $k_{o}$ (gmol.min ${ }^{-1} . \mathrm{cm}^{-}$ 
${ }^{2}$ ) e a energia de ativação da reação, $E_{a}\left(\mathrm{~kJ} \cdot \mathrm{mol}^{-1}\right)$.

$$
k=A \exp \left(\frac{E_{a}}{\mathcal{R} T}\right)
$$

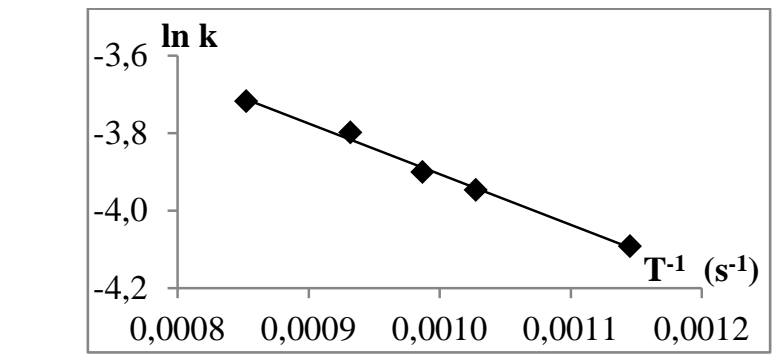

Figura 7 Efeito da temperatura sobre a reação $\mathrm{C}+\mathrm{O}_{2} \rightarrow \mathrm{CO}_{2}$.

A Figura 7 apresenta o gráfico onde a equação de Arrhenius linearizada foi plotada. Analisando o comportamento dos resultados, pode-se observar que há um ajuste satisfatório do modelo, indicando que nas temperaturas em que foram realizados os testes, trabalhou-se dentro do regime químico.

Portanto, para a energia de ativação obteve-se $10,8 \mathrm{~kJ} \cdot \mathrm{mol}^{-1}$ e 13,817 gmol.min ${ }^{-1} \cdot \mathrm{cm}^{-2}$ para o fator pré-exponencial. Já para a ordem da reação, os valores foram decrescentes ao longo da temperatura. Para a temperatura de $600{ }^{\circ} \mathrm{C}$ obteve-se uma ordem de 0,6 e para a temperatura de $900{ }^{\circ} \mathrm{C}$ a ordem obtida foi de 0,2 . Indicando que a medida que a Reação de Boudouard se faz presente é necessário considerar a concentração de $\mathrm{CO}_{2}$ para fins de cálculo.

\section{CONCLUSÕES}

Com base nos resultados preliminares obtidos nas análises realizadas em termobalança e o tratamento dos dados, se pode inferir que o modelo proposto é válido para o trabalho realizado. Para os cálculos utilizou-se o modelo do núcleo não reagido, onde se observou que há predomínio de controle cinético, com energia de ativação de $10,8 \mathrm{~kJ}_{\text {amol}}{ }^{-1}$ e fator préexponencial de 13,817 gmol.min ${ }^{-1} \cdot \mathrm{cm}^{-2}$. Observa-se que para o cálculo dos parâmetros cinéticos considerou-se apenas a reação $\mathrm{C}+\mathrm{O}_{2} \rightarrow \mathrm{CO}_{2}$. Este fenômeno ocorre em temperaturas inferiores à $720^{\circ} \mathrm{C}$. Entretanto, para temperaturas mais elevadas, além da reação de oxidação do char, também ocorre a reação de Boudouard $\mathrm{C}+\mathrm{CO}_{2} \rightarrow 2 \mathrm{CO}$. Esta consideração pode explicar a ordem da reação ser decrescente ao longo da temperatura.

\section{REFERÊNCIAS}

BEN - Balanço Energético Nacional 2013: Ano base 2012, Empresa de Pesquisa Energética. - Rio de Janeiro EPE, 2013.

DUAN, L., SUN, H., ZHAO, C., ZHOU, W., CHEN, X. Coal combustion characteristics on an oxy-fuel circulating fluidized bed combustor with warm flue gas recycle. Fuel, v. 127, p. 
$47-51,2014$.

IEA - International Energy Agency. $\mathrm{CO}_{2}$ emission from fuel combustion, França, 2013.

JOSÉ H. J. Zur Reaktivität von Koksen aus Santa Catarina-Steinkohle, Brasilien, bei der Vergasung mit Wasserdampf und Kohlendioxid. Thesis, RWTH Aachen, Germany, 1989.

KATHAMI, R; STIVERS, C.; JOSHI, K.; LEVENDIS, Y. A.; SAROFIM, A. F. Combustion behavior of single particles from three different coal ranks and from sugar cane bagasse in $\mathrm{O}_{2} / \mathrm{N}_{2}$ and $\mathrm{O}_{2} / \mathrm{CO}_{2}$ atmospheres. Combustion and Flame, v. 159, p. 1253-1271, 2012.

LECKNER, B., GOMÉS-BAREA, A. Oxy-fuel combustion in circulating fluidized bed boilers, Applied Energy, v. 125, p. 308 - 318, 2014.

LEVENSPIEL, O. Chemical Reaction Engineering, $3^{\mathrm{a}}$ ed., John Wiley \& Sons, Nova Iorque, 1976.

NUNES, K. G. P., MARCÍLIO, N. R. Determinação dos parâmetros cinéticos da reação de oxicombustão de carvão mineral. Dissertação de mestrado - Programa de pós-graduação em Engenharia Química - Universidade Federal do Rio Grande do Sul, 2012.

POHLMANN, J. G., OSÓRIO, E., VILELA, A. C. F., BORREGO, A. G. Reactivity to $\mathrm{CO}_{2}$ of chars prepared in $\mathrm{O}_{2} / \mathrm{N}_{2}$ and $\mathrm{O}_{2} / \mathrm{CO}_{2}$ mixtures for pulverized coal injection (PCI) in blast furnace in relation to char petrographic characteristics. International Journal of Coal Geology, v. 84, p. $293-300,2010$.

ROY, B. and BHATTACHARYA, S. Oxy-fuel fluidized bed combustion using Victorian brown coal: An experimental investigation. Fuel Processing Technology, v. 117, p 23-29, 2014.

SCHMAL, M.; MONTEIRO, J. L. F.; CASTELLAN, J. L. Kinetics of coal gasification, Ind. Eng. Chem. Process Des. Dev., 21, p 256 - 266, (1982).

TOFTEGAARD, M. B.; BRIX, J.; JENSEN, P. A.; GLARBORG, P.; JENSEN, A. D. Oxyfuel combustion of solid fuel, Progress. in Energy and Combustion. Science., v. 36, p. 581 - 625, 2010. 